\title{
El medio ambiente en un mundo globalizado y sus repercusiones en la salud física y emocional
}

The environment in a globalized world and its repercussions on physical and emotional health

Jarentzy Cruz Olvera $^{a}$

\begin{abstract}
:
Society has had great changes to date thanks to the development of industries and technologies, these have benefited the human being in an important way but also in a negative way. In this article these transformations are studied in the daily life of the people and the main repercussions of these in the health, to mention some, the emotional changes, the violence, the malnutrition, the chronic degenerative diseases and even respiratory diseases by the contamination of the environment For which a systematic review was carried out in PUBMED and the official website of the World Health Organization. Twenty articles were detected, of which 15 were selected, of which 7 focus on "The selection of food as a determinant of health" and 3 on "Environment and its relationship with human emotions" The results of this analysis allow establish that anthropogenic activity impacts not only the environmental environment, but also how people feel in their physical and emotional health.
\end{abstract}

Keywords:

environment, food, pollution, chronic-degenerative diseases, Obesity, emotional state

\begin{abstract}
Resumen:
La sociedad ha tenido hasta hoy grandes cambios gracias al desarrollo de industrias y de tecnologías, estos han beneficiado al ser humano de una manera importante pero también de manera negativa. En este artículo se estudian estas transformaciones en la vida cotidiana de las personas y las principales repercusiones de estas en la salud, por mencionar algunas, los cambios emocionales, la violencia, la desnutrición, las enfermedades crónico-degenerativas y hasta enfermedades respiratorias por la contaminación del ambiente. Para lo cual se llevó a cabo una revisión sistemática en PUBMED y la página oficial de la Organización Mundial de la Salud. Se detectó 20 artículos, de cuales se seleccionaron 15, de ellos 7 hacen hincapié en "La selección de alimentos como un factor determínate de la salud" y 3 en "Medio ambiente y su relación con las emociones humanas" Los resultados de este análisis permiten establecer que la actividad antropogénica impacta no solo en el entorno ambiental, sino en cómo se sienten las personas en su estado de salud físico y emocional.
\end{abstract}

Palabras Clave:

ambiente, alimentos, contaminación, enfermedades crónico-degenerativas, Obesidad, estado emocional

\section{Introducción}

A través de los años han surgido cambios que mantiene en constante desarrollo a la sociedad, estos a su vez pueden resultar muy favorables, pero así mismo tiempo pueden perjudicarlos.

Con el tiempo el hombre fue cambiando, sus formas de vida dejando atrás el amor por la naturaleza y poco a 'poco empezó a transformar su mundo en algo globalizado.

De acuerdo a diversos artículos se observó cómo hay un alto consumo de comida poco saludables (comida charra y rápida) que debido al horario cada individuo lo adapta a sus necesidades por lo que no siempre resulta beneficioso y entonces se presentan problemas que no deberían tener lugar haciendo a un lado su estado de salud.

\section{La selección de alimentos como un factor determinante de Salud física.}

Se le llama estado nutricional a la condición física que presenta una persona, como resultado del balance entre sus necesidades e ingesta de energía y nutrientes, la posibilidad de que un nutrimento participe en el metabolismo celular dependerá de su aparición en el medio interno.

Desafortunadamente cada vez la población tiene un estilo de vida menos saludable, es por eso que la mala alimentación empuja a la gente a tener sobrepeso.

\footnotetext{
${ }^{a}$ Estudiante de la Maestría en Salud Pública en Instituto Elise Freinet,Carretera La Paz No.442, Col. La Paz, C.P. 42092, Pachuca de Soto, Hidalgo. Autor de correspondencia / Jarentzy Cruz Olvera. Email: jarentzy@ hotmail.com
} 
Cuando se produce un desequilibrio entre las necesidades y la ingesta de energías y nutrientes, y esta situación se mantiene durante un periodo prolongado, se producen diversas enfermedades.

La ingesta de alimentos chatarra, aun cuando no es el único factor para la obesidad, se combina con el sedentarismo, hábitos alimentarios adquiridos dese los primeros años de vida y la culturas que se has conformado a lo largo de varias generaciones; por lo que has continuación se hará mención a las 10 consecuencias que provocan los alimentos insalubres:

1. Problemas de memoria y aprendizaje, Se menciona que consumir comida chatarra durante 5 días seguidos genera ciertas reacciones químicas que llevan a al inflamación del hipocampo, asociado a la memoria y reconocimiento.

2. Depresión entre los jóvenes, Las dietas saludables son fundamentales para mantener los cambios hormonales a raya.

3. Fatiga y debilidad.

4. Problemas digestivos. La falta de fibra obstaculiza la digestión y aumenta el riesgo de padecer hemorroides y constipación.

5. Aumenta el riesgo de Enfermedades Cardiovasculares.

Aumentan directamente los niveles de triglicéridos y colesterol malo lo que lleva a la formación de placas y enfermedades cardiovasculares.

6. Puede producir enfermedades renales.

Los niveles levados de grasas malas y el sodio de la sal afectan el balance sodio potasio generando hipertensión.

7. Daño en el hígado.

El consumo frecuente de comida chatarra genera un efecto dañino como el alcohol en el hígado.

8. Aumenta el riesgo de padecer cáncer.

El consumo excesivo de comida chatarra puede aumentar las probabilidades de padecer cáncer colorectal.

9. Puede causar diabetes mellitus tipo 2

Ya que la comida chatarra no contiene fibras con este tipo de comidas se lleva directamente al aumento en los niveles de azúcar en sangre.

10. Aumenta el riesgo de demencia

El exceso de comida basura y dulces puede aumentar sustancialmente los niveles de insulina en el cuerpo, el cerebro deja de responder a esta hormona y se vuelve resistente a ella. Esto puede restringir nuestra habilidad de razonar crear recuerdos, y por tanto aumentar el riesgo de padecer demencia.

Desafortunadamente el hombre ya ha llegado hasta un punto donde la globalización a sobrepasado todo, y que ahora existe un factor común en nuestras sociedades como lo es el hacinamiento.

También es muy notorio el contraste entre la vida urbana y la rural, que el ruido es un elemento que afecta nuestra sociedad; los medios de comunicación nos saturas día a día con anuncios que manipulan a las personas.

Otro punto importante es el crecimiento indiscriminado de las ciudades, lo que conlleva a que las zonas más lejanas o de la periferia de la ciudad este rodeada por poblaciones mu marginadas que viven en condiciones miserables y muy poco favorables para subsistir de buena manera tanto social como culturalmente.

Hoy en día se construyen múltiples torres de edificios separadas por pasillos impersonales, que promueven un ambiente no relacionado con las necesidades ni con los valores dela comunidad.

La basura que se produce hoy en día es mucho más toxica 'para el ambiente, debido a que ahora con este mundo globalizado se consumen más aparatos electrónicos que terminan dañando sumamente el ambiente.

\section{Metodología}

Se hizo una revisión sistemática y se recopilo 20 artículos que abordan el tema cambio ambiental con repercusiones en la salud de los cuales 15 resultaron ser útiles para la elaboración de este artículo.

\section{Resultados}

Las principales enfermedades observadas en un individuo afectado o por los cambios antes mencionados son:

$\begin{array}{ll}\text { - } & \text { Enfermedades cardiovasculares } \\ \text { - } & \text { Hipertensión arterial } \\ \text { - } & \text { Enfermedades coronarias } \\ \text { - } & \text { Cambios en el sistema digestivo } \\ \text { - } & \text { Trastornos de la defecación } \\ \text { - } & \text { Colitis } \\ \text { - } & \text { Gastritis } \\ \text { - } & \text { Depresión } \\ \text { - } & \text { Agresividad } \\ \text { - } & \text { Ansiedad } \\ \text { - } & \text { Violencia } \\ \text { - } & \text { Enfermedades respiratorias } \\ \text { - } & \text { Neumonías } \\ \text { - } & \text { Infecciones recurrentes }\end{array}$

\section{Discusión}

El mundo hoy en día se encuentra lleno de transformaciones que el mismo hombre ha generado un trabajo con horario prolongado, una ocupación estresante, fácil acceso a comida chatarra, sedentarismo, ausencia de tiempo libre o recreación, 
contaminación del aire, del agua y del suelo, desechos e industrias, son algunos de los problemas a los que el hombre moderno se tiene que enfrentar como consecuencia de su desarrollo económico.

Pero con el paso de los años se ha sufrido una transición epidemiologia, es decir, se dejaron atrás las muertes por infecciones y ahora las tasas de mortalidad están determinadas por enfermedades crónico degenerativas consecuencia de los cambios en la sociedad, el desgaste que sufren el ser humano por horarios prolongados, consumo de comida rápida, estrés y contaminación.

\section{Conclusión}

El medio ambiente definitivamente se encuentra asociado con el proceso salud enfermedad dependiendo de las condiciones ambientales en cuanto a contaminación y tipo de contaminantes que finalmente determinaran el tipo de enfermedad con mayor incidencia en la población humana.

\section{Referencias}

[1] Padilla Córdoba, D. A., Lazcano Melo, M. E., Moreno Uiostegui, D. A., Ramírez González, A., Imbert Palafox, J. L., Molina Trinidad, E. M., López Contreras L., Ruvalcaba Ledezma, J. C. (2018). El medio ambiente en un mundo globalizado y sus repercusiones en la salud física y emocional. Educación y Salud Boletín Científico de Ciencias de la Salud del ICSa, 7(13) 99-104. 